\title{
The role of extracellular vesicles in modulating the host immune response during parasitic infections
}

\section{Sergio Montaner ${ }^{1}$, Alicia Galiano ${ }^{1}$, María Trelis ${ }^{1}$, Lorena Martin-Jaular ${ }^{2}$, Hernando A. del Portillo ${ }^{2,3}$, Dolores Bernal ${ }^{4}$ and Antonio Marcilla ${ }^{1 *}$}

1 Àrea de Parasitologia, Departament de Biologia Cel.lular i Parasitologia, Universitat de València, Burjassot, Spain

2 Barcelona Centre for International Health Research (CRESIB, Hospital Clínic-Universitat de Barcelona), Barcelona, Spain

${ }^{3}$ Institució Catalana de Recerca i Estudis Avançats, Barcelona, Spain

${ }^{4}$ Departament de Bioquímica i Biologia Molecular, Universitat de València, Burjassot, Spain

\section{Edited by:}

Martin Johannes Hoogduijn, Erasmus Medical Center, Netherlands

Reviewed by:

Urszula Krzych, Walter Reed Army Institute of Research, USA

Olivier Silvie, Institut National de la

Santé et de la Recherche Médicale,

France

*Correspondence:

Antonio Marcilla, Av. Vicent Andrés

Estellés s/n, 46100 Burjassot,

Valencia, Spain

e-mail: antonio.marcilla@uv.es
Parasites are the cause of major diseases affecting billions of people. As the inflictions caused by these parasites affect mainly developing countries, they are considered as neglected diseases. These parasitic infections are often chronic and lead to significant immunomodulation of the host immune response by the parasite, which could benefit both the parasite and the host and are the result of millions of years of co-evolution. The description of parasite extracellular vesicles (EVs) in protozoa and helminths suggests that they may play an important role in host-parasite communication. In this review, recent studies on parasitic (protozoa and helminths) EVs are presented and their potential use as novel therapeutical approaches is discussed.

Keywords: extracellular vesicles, parasite, protozoa, helminth, immunomodulation

\section{PARASITIC DISEASES AND EXTRACELLULAR VESICLES}

Evidence of parasite infections has been found very early in human evolution. In fact, some parasites were inherited from our primate ancestors in Africa, and some others were acquired from animals during our evolution, migrations, and agricultural practices (1). It is estimated that about 300 species of helminths and over 70 species of protozoa affect humans (1). A relatively small proportion of these parasites cause some of the most important diseases in the world, such as malaria, Chagas' disease, sleeping sickness, schistosomiasis, filariasis, and soil-transmitted helminthiasis among others. Despite their elevated global prevalence, they are considered as neglected tropical diseases ${ }^{1}$. In endemic areas, epidemiological studies of some immunological disorders (i.e., atopy) suggest that current parasitic infections have a protective effect (2). In contrast, in developed countries, where improved living conditions and vaccination are common, the lack of exposure of our immune system to infections of historical importance, could lead to an increase in hypersensibility and autoimmune diseases.

In the last decades, extracellular vesicles (EVs) have been well recognized as mediators of intercellular communications in prokaryotes and eukaryotes. They are able to carry proteins, lipids, and nucleic acids, which are incorporated by recipient cells, where in turn they have different effects. EVs carry a common group of proteins and also specific proteins that reflect the particular role and/or composition of their cell of origin. EVs include usually apoptotic bodies, microparticles/microvesicles (originated by plasma membrane budding), and exosomes [released from multivesicular bodies (MVBs)] (3, 4). Different EVs have been described in most groups of parasitic protozoa, including

${ }^{1}$ http://www.who.int/neglected_diseases/diseases/en/ flagellates, sporozoa, and microsporidians, and they have been detected in extracellular and intracellular stages. In addition to protozoa, parasitic helminths have been recently shown to release EVs (Table 1).

With respect to EVs composition, studies in Leishmania spp. parasites, the protozoan causing different forms of leishmaniases ${ }^{2}$, have shown the presence of protein homologs to known proteins that regulate exosome biogenesis and release in mammalian cells $(42,43)$. Recently, Silverman and Reiner have proposed that Leishmania are capable of secreting both exosomes and plasma membrane blebs, as mammalian cells do, suggesting that both types could play a role in pathogenesis (5). Most of the studies on EVs composition have been focused on intracellular stages of Leishmania spp., and it has been shown that changes in the environment seem to affect vesicle release and cargo $(6,42)$. In fact, proteomic analysis has revealed that the protein cargo of Leishmania exosomes is quantitative different in response to changes in temperature and $\mathrm{pH}(42)$. In this context, exosomes obtained at neutral $\mathrm{pH}$ were enriched in kinase activity, meanwhile in acidic $\mathrm{pH}$ they were enriched in phosphatase activity (42). Similar results were obtained after treating in vitro extracellular stages of Leishmania with a short heat-shock treatment (6).

Among the proteins identified in Leishmania EVs, there are virulence factors like GP63/leishmanolysin, membrane proteins, and redox enzymes like tryparedoxin peroxidase and heat-shock proteins (Hsp) (5). As suggested by Silverman and Reiner, this specific packaging of individual proteins and functional groups may likely reflect a sophisticated packaging of virulence factors by Leishmania in response to specific environments (5). 


\section{PROTOZOANS}

\section{Trypanosomatids}

\section{Leishmania spp.}

Trypanosoma brucei

Trypanosoma cruzi

Apicomplexa (sporozoa)

Plasmodium vivax

Plasmodium berghei

Plasmodium yoelii

Plasmodium falciparum

Plasmodium malariae

Toxoplasma gondii

Cryptosporidium parvum

Eimeria spp.

\section{Flagellates}

Trichomonas vaginalis

Giardia duodenalis

\section{HELMINTHS}

\section{Cestodes}

Echinococcus multilocularis

Trematodes

Schistosoma spp.

Echinostoma caproni

Fasciola hepatica

Dicrocoelium dendriticum

Nematodes

Heligmosomoides polygyrus
Exosomes and EVs from infected macrophages

(5-8)

Exosomes

Outer membrane-derived vesicles, exosomes

(9)

(9-15)

Plasma-derived MPs

$(16,17)$

Plasma-derived MPs (from infected mice)

$(18,19)$

Plasma-derived exosomes

(20)

Plasma-derived exosomes and vesicles $(60-100 \mathrm{~nm})$ and microvesicles (100-1000 nm) (17, 21-23)

Plasma-derived exosomes

(17)

Exosomes from infected cells; exosomes

(24-26)

Exosomes from infected cells

Dendritic cells derived exosomes (from infected chickens)

(27)

$(28,29)$

Exosomes

(30)

Secretory vesicles, exosomes

$(31,32)$

Vesicles derived from metacestodes

(33-35)

Shedding vesicles

(36)

EVs; exosomes

$(37,38)$

EVs; exosomes

$(37,39)$

Exosomes

(40)

EVs
(41)

Data were obtained also from Ref. not cited in the text $(8,9,14,15,19,22,23,33-36,38,39)$

Other intracellular organisms like apicomplexans Plasmodium and Toxoplasma species have been described to produce EVs.

Plasmodium species are the causative agents of malaria, a disease affecting an estimated 207 million individuals ${ }^{3}$. Although previous studies had detected EVs in peripheral blood of Plasmodium falciparum as well as Plasmodium vivax patients (16-18), the first description of Plasmodium spp. exosomes was reported in 2011 by Del Portillo and co-workers (20), who revealed the presence of parasite proteins in reticulocyte-derived exosomes (rex) from experimental infections. These parasite antigens included serine-repeat antigens, merozoite surface proteins 1 and 9, metabolic enzymes like lactate dehydrogenase, GAPDH, enolase and aldolase, cysteine proteases, and Hsp among others (20).

Toxoplasma gondii is responsible for toxoplasmosis, an important public health problem infecting about $30 \%$ of the world's population, including immunocompromised individuals (44). Toxoplasma is promiscuous and can infect virtually any nucleated host cell (45). The existence of EVs ( $65 \mathrm{~nm}$ ) in MVBs, has been detected in Toxoplasma secretory organelles (24). Lately, the presence of miRNA in T. gondii exosomes has been reported (25).

${ }^{3}$ http://www.who.int/mediacentre/news/releases/2013/world-malaria-report20131211/en/
The kinetoplastida Trypanosoma cruzi and Trypanosoma brucei are the causal agents of the Chagas' disease and sleeping sickness, respectively. Chagas' disease affects $7-8$ million people mostly in Latin America ${ }^{4}$, and sleeping sickness threats millions of people in 36 countries in sub-Saharan Africa ${ }^{5}$.

The extracellular phase of T. cruzi (trypomastigota) produces EVs that contain surface components like glycoproteins gp85/transialidases, alphaGal-containing molecules, proteases (i.e., cruzipain), cytoskeleton proteins, mucins, and associated to GPI (glycosylphospatidylinositol)-anchored molecules. All these molecules are engulfed by host cells in the absence of parasitic cells, and are accumulated in phagocytic/endocytic compartments (10).

In addition to proteins, the presence of small RNA in EVs from T. cruzi has been reported, including tRNA, which were actively secreted to the extracellular medium and acted as vehicle for the transfer of these molecules to other parasites and to mammalian cells (11). Furthermore, EVs secreted by T. cruzi epimastigotes are able to induce epigenetic changes in host cells (12).

The extracellular flagellate Trichomonas vaginalis is the causal agent of trichomoniases, the most prevalent curable sexually

\footnotetext{
${ }^{4}$ http://www.who.int/mediacentre/factsheets/fs340/en/

${ }^{5} \mathrm{http}: / /$ www.who.int/mediacentre/factsheets/fs259/en/
} 
transmitted infection globally (46). This parasite produces EVs to allow its attachment to the host mucosa (30). The proteomic analyses of these EVs revealed that $75 \%$ of the identified proteins corresponded to orthologs of mammalian exosome proteomes (exocarta). Common proteins represent core conserved exosomes protein families such as tetraspanins, Alix, Rabs, Hsp70, subunits of heterotrimeric G proteins, and TcTP (30). The identified proteins were sorted into functional groups, and the more abundant corresponded to signaling proteins (14\%), metabolic enzymes (14\%), cytoskeletal proteins, and proteins involved in transport and vacuolar proteins (30).

When comparing the proteomics profiles of intracellular and extracellular protozoa EVs, it seems that a common pattern of proteins is present in both, which include metabolic enzymes and Hsp. An enrichment in proteins involved in transport and vacuolar proteins is observed in EVs from extracellular protozoa (i.e., tetraspanins).

The diseases caused by helminths are considered the most neglected ones, with a third of the human population affected at least by one species (47). EVs from the trematode species Echinostoma caproni, Dicrocoelium dendriticum, and Fasciola hepatica have been isolated. The analysis of the composition of these vesicles has identified proteins previously described in the excretory/secretory products (ESP) (about $50 \%$ of proteins corresponded to the proteins identified in the secretome) $(37,40)$, which may explain the atypical protein secretion (lacking typical secretion signals) in flukes. These proteins include metabolic enzymes like enolase, GAPDH, aldolase, and well-known exosome components like Hsp70 and annexins (37, 40). Differences in EVs composition were observed among the three species, correlated with their respective ESP. Meanwhile, no proteases were present in E. caproni EVs, D. dendriticum, and F. hepatica EVs contained leucine aminopeptidase (LAP), and F. hepatica EVs contained a large number of proteases (i.e., cathepsins), probably related to its migration along tissues $(37,40)$, as well as chaperons, fatty-acid binding proteins, and detoxifying enzymes (37). In addition to the presence of proteins in helminth EVs, the presence of miRNA in D. dendriticum EVs has been described (40).

\section{PARASITIC EVS IN CELL-CELL COMMUNICATION}

Parasite EVs participate in parasite-parasite and host-parasite communication processes.

Very little information is available about the role of EVs in intraspecific communications. A recent study has demonstrated that $P$. falciparum infecting red blood cells directly communicates with other parasites using EVs that are capable of delivering genes. Importantly, communication via EVs also promotes differentiation to sexual forms and survival of parasites, providing a mechanism for increasing parasite persistence in times of stress (48). Furthermore, the P. falciparum PfEMP1 trafficking protein (PfPTP2), which plays a key role in the traffic to host cells, has been identified. PfPTP2 functions in the release of EVs into the supernatant, implicating $P$. falciparum molecular machinery in intercellular communications (48).

In contrast, there are many examples of parasite EVs involved in host-parasite communication.
Pioneering studies on EVs in trypanosomes described T. cruzi shedding vesicles $(20-80 \mathrm{~nm})$ in cultured trypomastigotes (13), and recently, a possible association between intensity of shedding and infectivity of different strains has been proposed (47). These authors suggest that these vesicles could be acting as messengers for invasion, somehow preparing the host cell for the incoming trypanosome, which represents a novel mechanism to explain parasite interaction with the host $(10,49)$.

Various studies have described the isolation of EVs from different $T$. cruzi stages $(10,11,26,49)$. Importantly, the preimmunization of mice using trypomastigote vesicles induce severe heart pathology with intense inflammatory reaction and higher number of amastigote nests in cardiac tissue (10), indicating the impact of host-parasite communication. After EVs release, these vesicles form a complex with C3 convertase on the parasite surface, stabilizing the enzyme and inhibiting its activity, protecting parasites from complement lysis and increasing parasite survival (50). Interestingly, these vesicles also carry transforming growth factor $\beta$ (TGF $\beta$ ), which could promote parasite invasion in the course of infection in vivo and in vitro (50).

As pointed out by Deolindo et al. (26), the production of EVs by infective stages of $T$. cruzi confirms their role in parasite survival strategies and in cell-cell communication. Supporting this notion, Garcia-Silva et al. (12) have shown that parasite EVs elicited changes in the host transcriptome upon their incorporation in the cells, which include modification of immune responses pathways (12).

Little is known about EVs in Giardia duodenalis, an extracellular parasite of the human intestine that causes diarrheal illness, and with high global prevalence 6 . Some authors reported the presence of secretory vesicles in this parasite associated with encystation processes. The process of release of these vesicles has been suggested to occur after fragmentation of large encystation-specific secretory vesicle in small secretory vesicles, followed by exocytosis, but this was not fully demonstrated $(31,32)$. Because Giardia is one of the earliest branching protists, knowledge of the secretory organelle biogenesis that occurs during its differentiation into cysts offers novel insights into the molecular machinery required for the regulation of the protein transport in higher organisms $(31,32)$. Recently, it has been reported an increase in G. duodenalis EVs formation in response to different conditions (i.e., $\mathrm{pH}$ changes, presence of bile, etc.), suggesting that these vesicles could provide a mechanism for parasite adaptation to changing environment encountered in the host during the course of the infection (26).

The finding that helminth EVs are internalized by host cells suggests an important role for these vesicles in host-parasite communications (37). It is possible that helminths could send messengers like mRNA or miRNA into EVs to act on host targets. Supporting this notion, the presence of molecules of miRNA in D. dendriticum EVs has been reported (40). Preliminary studies have shown that the nematode Heligmosomoides polygyrus produce EVs, which alter inflammatory responses in both cultured cells and in a murine model. These findings would explain how these nematode EVs could mediate cross-phylum communication and may help to suppress the host inflammatory response (51).

\footnotetext{
${ }^{6}$ http://www.cdc.gov/parasites/giardia/epi.html\#one
} 


\section{INDUCTION OF HOST EVs SECRETION}

Some descriptions of host-parasite communication through EVs in infected cells have been reported, with examples including mainly apicomplexan like Toxoplasma (27), Plasmodium (16-18), Eimeria $(28,29)$, and Cryptosporidium (52) species.

As mentioned above, Plasmodium proteins were detected in rex in experimental infections, which confirm that they are taken up by host cells (20). Studies to determine whether these vesicles are constitutively released, or whether they are released during a particular phase in the parasite cycle, have been reported recently (21). They present evidence that reticulocyte microvesicles (RMVs) release increases steadily during the parasite cycle and peaks late during schizogony or shortly thereafter. This pattern of release coincides with the emergence of a prominent vesicular subpopulation of $150-250 \mathrm{~nm}$ in the infected red blood cells (iRBCs) preparation. Altogether, these data demonstrate that the peak release of RMVs from iRBCs occurs shortly before egression (i.e., within the last 6-8 h of the parasite asexual cycle) (21).

Another apicomplexan protozoan are Eimeria spp., the etiologic agents of avian coccidiosis, a major parasitic disease of poultry (28). EVs from dendritic cells infected with Eimeria tenella parasites were shown to protect animals by (a) increasing body weight gain, (b) decreasing feed conversion ratios, (c) reducing fecal oocyst shedding, (d) decreasing intestinal lesions, and (e) reducing mortality compared with animals given parasite $\mathrm{Ag}$ alone (28). Similar results were obtained for other Eimeria species, suggesting that this protocol is an efficient way of immunizing against other apicomplexans (29).

Cryptosporidium species are another example in which intracellular parasites communicate with their host increasing the production of EVs. Cryptosporidiosis is one of the most frequent causes of diarrhea worldwide, affecting immunocompromised and/or immunocompetent patients (53). The occurrence of largescale outbreaks of human cryptosporidiosis is often attributed to contaminated drinking water (54).

It has been reported that Cryptosporidium parvum infection increases luminal release of EVs from the biliary epithelium, probably through TLR4/IKK2-mediated activation of the multivesicular body exocytic pathway (52). Immunogold staining revealed that these microvesicles were positive for the exosome markers CD63 and ICAM-1. Release of EVs involves activation of TLR4/IKK2 signaling through promoting the SNAP23-associated vesicular exocytic process (52). Furthermore, these authors presented evidence that activation of TLR4 signaling stimulates the biogenesis and luminal release of antimicrobial peptide-shuttling EVs. The anti-C. parvum activity of apical EVs released from the epithelium may involve direct binding these vesicles to the C. parvum sporozoite surface (52). Confocal analyses showed the fusion of these EVs with C. parvum sporozoites causing cargo release within the parasite. These results suggest that all extracellular stages of C. parvum (sporozoites, merozoites, and microgametocytes) may be vulnerable to EVs binding/targeting, contributing to gastrointestinal mucosal anti-C. parvum defense (52).

Extracellular vesicles derived from macrophages infected with Leishmania mexicana display unique protein signatures (composition and abundance of many functional families of proteins, such as plasma membrane-associated proteins, chaperones, and metabolic enzymes) (7). L. mexicana surface protease GP63 has been identified in EVs from macrophages exposed to parasite promastigotes (7).

\section{PARASITIC EVs AS IMIMUNOMODULATORS}

Many of the immunomodulatory proteins lack typical secretion signals for delivery to the extracellular environment, so new secretion routes should be active, involving host-parasite interactions at cellular and subcellular levels, which in turn could be related to immunomodulation processes.

The role of exosomes in modulating the immune response was first described in Leishmania spp. in 2010 (42). This study also demonstrated that certain factors associated with infection were able to positively regulate the release of exosomes and modulate their composition (42). A recent study has shown that Leishmania parasites mutants lacking the metalloprotease GP63 have a reduced modulatory capacity in relation to wild-type parasites in animal models, and they have also described that exosomes are involved in recruiting neutrophils exhibiting stronger pro-inflammatory properties than the neutrophils recruited by parasites (55).

Trypanosomes also constitute a good example of immunomodulation. Proteins like phosphoglycerate mutase, enolase, pyruvate kinase, and phosphoglycerate kinase, known to be involved in immunosuppressive activity in other organisms, have been also found in the T. brucei secretome, suggesting a similar role in infection. T. brucei releases a higher amount of proteins in EVs than using a classical secretory pathway, which suggests that T. brucei may deliver an avalanche of new epitopes to overwhelm the host immune system, or to communicate between trypanosomes themselves. This is achieved by exchanging receptors in their cytosolic form, which may represent an important survival strategy at the population level (9). Several proteins secreted by T. brucei are also detected in Leishmania and T. cruzi secretomes, suggesting that they are the result of an active and common secretion process (49).

They are interesting immunomodulation studies in malaria. When mice were immunized with Plasmodium yoelii purified rex, an increase in the reticulocytemia and in the production of IgG antibodies capable of recognizing $P$. yoelii iRBCs was observed. Remarkably, in combination with the adjuvant $\mathrm{CpG}$ oligodeoxynucleotide, rex from an experimental infection with a $P$. yoelii reticulocyte-prone non-lethal strain conferred full and long-lasting protection upon immunization and lethal challenge. Thus, these data show, for the first time, that rex can be explored as a new vaccine against malaria (20).

Furthermore, Mantel et al. have demonstrated that the release of RMVs from iRBCs can activate the pro-inflammatory cytokines interleukin-6 (IL-6), IL-12, and IL-1b, as well as the antiinflammatory cytokine IL-10, in a dose-dependent manner (21). They also showed that neutrophils pre-incubated with RMVs from uninfected red blood cells (uRBCs) migrated at a slower rate compared these pre-incubated with RMVs from iRBCs or untreated controls. Together, these data demonstrate that RMVs from iRBCs, but not from uRBCs, can strongly stimulate cells of the innate immune system (21).

Extracellular parasites could use exosomes to deliver proteins and/or RNA to manipulate host cell responses, while remaining 


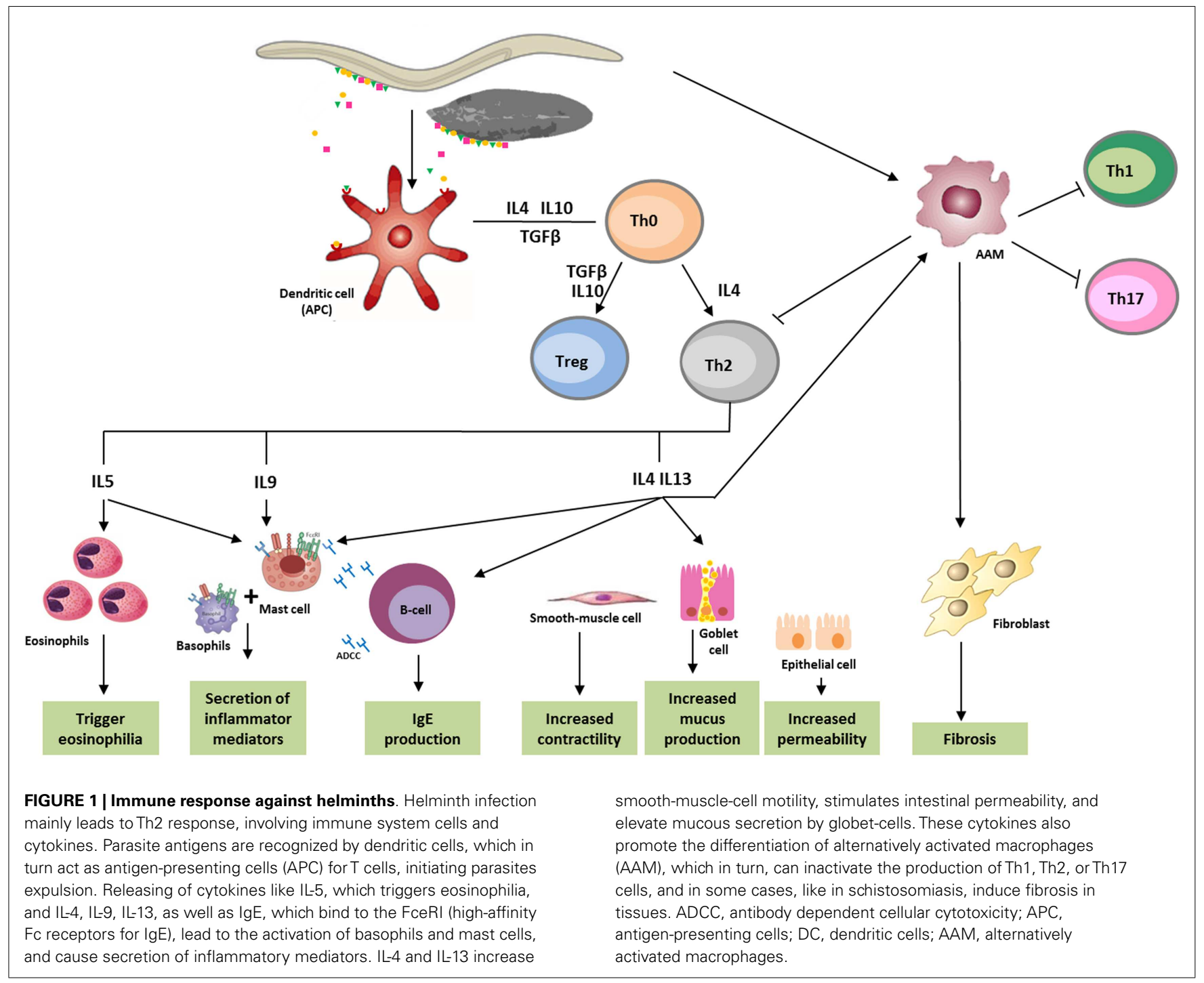

in the extracellular space, generating important changes in both host immune response and parasite attachment to host cells (30). In this context, $T$. vaginalis may use exosomes to manipulate host defense responses, similarly to the secretion of virulence factors and vesicles by pathogenic bacteria (30). By reducing the IL- 8 expression of host ectocervical cells, T. vaginalis exosomes may be playing an important role in the establishment of a chronic infection. These EVs may lead to the regulation of IL-6 and IL-8 secretion, preparing and facilitating colonization of the urogenital tract (30).

Helminthiases are parasitic diseases with a high prevalence, which reflects their ability to manipulate the host immune system, preventing parasite expulsion. Helminths are interesting organisms to study immunomodulation, since host immunity has also developed mechanisms to limit their pathology and the ensuing injury, as in some cases, their elimination originates even worse collateral damage.

Immune responses to helminths comprise a combination of both innate defense and $\mathrm{Th} 2$ response, which disable, degrade, and dislodge the parasites (56). Characteristic features of helminth infection are Th2-dominated immune responses and Th1/Th17 immunity blocking, allowing for the survival of the parasite in a “modified Th2 environment" $(57,58)$ (Figure 1).

The immune regulation originated by helminths may offer new routes to treat immune dysfunctions like allergy, autoimmunity, and inflammatory bowel diseases $(58,59)$. Various clinical trials that use helminths to treat autoimmune diseases are underway (60). Furthermore, enhanced allograft tolerance with helminth infection has been reported in various species, suggesting that the infection or defined products from immunomodulatory helminths could be of interest in future transplantation protocols (61). Much research has focused on ESP released by live helminths, which can interfere with every aspect of host immunity. Schistosoma species secrete proteins capable of activating the release of IL-4 and activate the degranulation of basophils (human and mice) to promote a Th2 response in the surrounding environment. In addition, Schistosoma spp. eggs secrete among others, the protein omega-1, an abundant ribonuclease associated with egg transit 
through host tissues, and responsible for activating Th2 mechanisms that allow for egg survival and excretion. Furthermore, extensive glycosylation of some Schistosoma proteins also trigger Th2 responses in vivo through TLR4 ligation $(57,62)$.

Recent studies have shown that antigens derived from $F$. hepatica tegument inhibit mast cells, which normally play a protective role during microbial infections. This modulating effect is mediated by the induction of suppressors of cytokine signaling (SOCS), which are essential for self-regulatory inflammatory Th1-dependent processes (63).

Interestingly, other helminths produce molecules that have a cytokine-like effect on mammalian cells. Proteins like the macrophage migration inhibitory factor (MIF) are produced by the nematode Brugia malayi (57). This parasitic molecule can synergize with IL-4 to induce the development of fully suppressive, alternatively activated macrophages in vitro. Thus, in a Th2 environment, parasitic MIF may prevent the classical pro-inflammatory activation of macrophages (57).

As reviewed by Dalton et al. (64), F. hepatica ESP includes molecules that drive the immune response toward a favorable, nonprotective, Th2-mediated environment. These immunomodulatory molecules include cathepsin L, peroxiredoxins, and helminth defense molecules (i.e., HDM-1/MF6p), which could help treat autoimmune diseases and chronic inflammation in humans and animals (64). Interestingly, two of these proteins (peroxiredoxins and cathepsins) are present in F. hepatica EVs (37), and the third type of immunomodulating molecules (HDM$1 /$ MF6p) have been detected in exosomes from the related trematode species D. dendriticum (40), and preliminary results suggest their presence in F. hepatica and E. caproni EVs (unpublished data).

Fasciola hepatica HDM-1/MF6p exhibits biochemical and functional characteristics similar to human defense peptides, particularly CAP18. FhHDM-1 modulates innate cell activation by classical toll-like receptor (TLR) ligands, such as lipopolysaccharide (LPS), indicating its therapeutic potential for autoimmune diseases (65). Furthermore, FhHDM-1 might mitigate the inflammatory response of macrophages to LPS by inhibiting the production of TNF $\alpha$ and IL- $1 \beta$, as mice treated with a single dose of FhHDM-1/MF6p prior to, or after, bacterial LPS had significantly lower levels of circulating TNF $\alpha$ and IL-1 $\beta$ (64-66). FhHDM$1 /$ MF6p has been recently characterized as a heme-binding protein, suggesting that its role as a heme chaperone that may participate in important physiological processes for the parasite (i.e., heme trafficking and storage). However, it does not seem to act as a primary ligand for LPS (67).

It has been shown that $F$. hepatica ESP prevents type 1 diabetes (T1D) in non-obese diabetic (NOD) mice, which is associated with suppression of IFN $\gamma$ secretion from auto-reactive T cells, and the switch to IgG1 auto-antibody production (68).

As previously mentioned, recent studies have shown the immunomodulatory effect $H$. polygyrus EVs on a murine model (51), confirming previous results with ESP from the same nematode (41).

Future studies will focus on the potential use of parasitic EVs as therapeutic tools to treat autoimmune disorders and chronic inflammation.

\section{CONCLUDING REMARKS}

There has been an increasing number of research publications dealing with the study of EVs and their role in intercellular communication and immunomodulation in the last few years. EVs have been described in parasitic organisms, mostly protozoa, and more recently in helminths. Parasitic protozoa EVs carry virulence factors, immunomodulatory molecules, and nucleic acids. These vesicles have been shown to provide long-lasting protection upon immunization and lethal challenge. Current clinical trials are evaluating the use of helminth secretory products to treat chronic inflammatory and autoimmune diseases. Interestingly, some of the characterized parasitic immunomodulatory proteins have been identified in EVs, raising the intriguing possibility of the therapeutic use of parasitic EVs.

\section{ACKNOWLEDGMENTS}

Dr. Lynne Yenush is acknowledged for critically reading the manuscript. Dr. Hernando A. del Portillo's and Marcilla's labs are members of the COST Action BM1202 "European Network on microvesicles and exosomes in health and disease."

\section{REFERENCES}

1. Cox FE. History of human parasitology. Clin Microbiol Rev (2002) 15:595-612. doi:10.1128/CMR.15.4.595-612.2002

2. Feary J, Britton J, Leonardi-Bee J. Atopy and current intestinal parasite infection: a systematic review and meta-analysis. Allergy (2011) 66:569-78. doi:10.1111/j. 1398-9995.2010.02512.x

3. Raposo G, Stoorvogel W. Extracellular vesicles: exosomes, microvesicles, and friends. J Cell Biol (2013) 200:373-83. doi:10.1083/jcb.201211138

4. Witwer KW, Buzas EI, Bemis LT, Bora A, Lasser C, Lotvall J, et al. Standardization of sample collection, isolation and analysis methods in extracellular vesicle research. J Extracell Vesicles (2013) 2:20360. doi:10.3402/jev.v2i0.20360

5. Silverman JM, Reiner NE. Leishmania exosomes deliver preemptive strikes to create an environment permissive for early infection. Front Cell Infect Microbiol (2012) 1:26. doi:10.3389/fcimb.2011.00026

6. Hassani K, Antoniak E, Jardim A, Olivier M. Temperature-induced protein secretion by Leishmania mexicana modulates macrophage signalling and function. PLoS One (2011) 6:e18724. doi:10.1371/journal.pone.0018724

7. Hassani K, Olivier M. Immunomodulatory impact of Leishmania-induced macrophage exosomes: a comparative proteomic and functional analysis. PLoS Negl Trop Dis (2013) 7:e2185. doi:10.1371/journal.pntd.0002185

8. Figuera L, Acosta H, Gomez-Arreaza A, Davila-Vera D, Balza-Quintero A, Quinones W, et al. Plasminogen binding proteins in secreted membrane vesicles of Leishmania mexicana. Mol Biochem Parasitol (2013) 187:14-20. doi:10.1016/ j.molbiopara.2012.11.002

9. Geiger A, Hirtz C, Becue T, Bellard E, Centeno D, Gargani D, et al. Exocytosis and protein secretion in Trypanosoma. BMC Microbiol (2010) 10:20. doi:10.1186/1471-2180-10-20

10. Trocoli Torrecilhas AC, Tonelli RR, Pavanelli WR, da Silva JS, Schumacher RI, de Souza W, et al. Trypanosoma cruzi: parasite shed vesicles increase heart parasitism and generate an intense inflammatory response. Microbes Infect (2009) 11:29-39. doi:10.1016/j.micinf.2008.10.003

11. Garcia-Silva MR, das Neves RF, Cabrera-Cabrera F, Sanguinetti J, Medeiros LC, Robello C, et al. Extracellular vesicles shed by Trypanosoma cruzi are linked to small RNA pathways, life cycle regulation, and susceptibility to infection of mammalian cells. Parasitol Res (2014) 113:285-304. doi:10.1007/s00436-013-3655-1

12. Garcia-Silva MR, Cabrera-Cabrera F, das Neves RF, Souto-Padron T, de Souza W, Cayota A. Gene expression changes induced by Trypanosoma cruzi shed microvesicles in mammalian host cells: relevance of tRNA-derived halves. Biomed Res Int (2014) 2014:305239. doi:10.1155/2014/305239

13. Goncalves MF, Umezawa ES, Katzin AM, de Souza W, Alves MJ, Zingales B, et al. Trypanosoma cruzi: shedding of surface antigens as membrane vesicles. Exp Parasitol (1991) 72:43-53. doi:10.1016/0014-4894(91)90119-H

14. Ouaissi A, Aguirre T, Plumas-Marty B, Piras M, Schoneck R, Gras-Masse H, et al. Cloning and sequencing of a $24-\mathrm{kDa}$ Trypanosoma cruzi specific antigen 
released in association with membrane vesicles and defined by a monoclonal antibody. Biol Cell (1992) 75:11-7. doi:10.1016/0248-4900(92)90119-L

15. Bayer-Santos E, Aguilar-Bonavides C, Rodrigues SP, Cordero EM, Marques AF, Varela-Ramirez A, et al. Proteomic analysis of Trypanosoma cruzi secretome: characterization of two populations of extracellular vesicles and soluble proteins. J Proteome Res (2013) 12:883-97. doi:10.1021/pr300947g

16. Campos FM, Franklin BS, Teixeira-Carvalho A, Filho AL, de Paula SC, Fontes CJ, et al. Augmented plasma microparticles during acute Plasmodium vivax infection. Malar J (2010) 9:327. doi:10.1186/1475-2875-9-327

17. Nantakomol D, Dondorp AM, Krudsood S, Udomsangpetch R, Pattanapanyasat $\mathrm{K}$, Combes V, et al. Circulating red cell-derived microparticles in human malaria. J Infect Dis (2011) 203:700-6. doi:10.1093/infdis/jiq104

18. Couper KN, Barnes T, Hafalla JC, Combes V, Ryffel B, Secher T, et al. Parasitederived plasma microparticles contribute significantly to malaria infectioninduced inflammation through potent macrophage stimulation. PLoS Pathog (2010) 6:e1000744. doi:10.1371/journal.ppat.1000744

19. Schrevel J, Asfaux-Foucher G, Hopkins JM, Robert V, Bourgouin C, Prensier G, et al. Vesicle trafficking during sporozoite development in Plasmodium berghei: ultrastructural evidence for a novel trafficking mechanism. Parasitology (2008) 135:1-12. doi:10.1017/S0031182007003629

20. Martin-Jaular L, Nakayasu ES, Ferrer M, Almeida IC, Del Portillo HA. Exosomes from Plasmodium yoelii-infected reticulocytes protect mice from lethal infections. PLoS One (2011) 6:e26588. doi:10.1371/journal.pone. 0026588

21. Mantel PY, Hoang AN, Goldowitz I, Potashnikova D, Hamza B, Vorobjev I, et al. Malaria-infected erythrocyte-derived microvesicles mediate cellular communication within the parasite population and with the host immune system. Cell Host Microbe (2013) 13:521-34. doi:10.1016/j.chom.2013.04.009

22. Trelka DP, Schneider TG, Reeder JC, Taraschi TF. Evidence for vesicle-mediated trafficking of parasite proteins to the host cell cytosol and erythrocyte surface membrane in Plasmodium falciparum infected erythrocytes. Mol Biochem Parasitol (2000) 106:131-45. doi:10.1016/S0166-6851(99)00207-8

23. Bhattacharjee S, van Ooij C, Balu B, Adams JH, Haldar K. Maurer's clefts of Plasmodium falciparum are secretory organelles that concentrate virulence protein reporters for delivery to the host erythrocyte. Blood (2008) 111:2418-26. doi:10.1182/blood-2007-09-115279

24. Torres M, Ducournau C, Dimier-Poisson I. Toxoplasma gondii: qualified to secrete exosomes? J Extracell Vesicles (2012) 1:18178. doi:10.3402/jev.vli0.18178

25. Pope SM, Lasser C. Toxoplasma gondii infection of fibroblasts causes the production of exosome-like vesicles containing a unique array of mRNA and miRNA transcripts compared to serum starvation. J Extracell Vesicles (2013) 2:22484. doi:10.3402/jev.v2i0.22484

26. Deolindo P, Evans-Osses I, Ramirez MI. Microvesicles and exosomes as vehicles between protozoan and host cell communication. Biochem Soc Trans (2013) 41:252-7. doi:10.1042/BST20120217

27. Bhatnagar S, Shinagawa K, Castellino FJ, Schorey JS. Exosomes released from macrophages infected with intracellular pathogens stimulate a proinflammatory response in vitro and in vivo. Blood (2007) 110:3234-44. doi:10.1182/blood2007-03-079152

28. Del Cacho E, Gallego M, Lee SH, Lillehoj HS, Quilez J, Lillehoj EP, et al. Induction of protective immunity against Eimeria tenella infection using antigen-loaded dendritic cells (DC) and DC-derived exosomes. Vaccine (2011) 29:3818-25. doi:10.1016/j.vaccine.2011.03.022

29. del Cacho E, Gallego M, Lee SH, Lillehoj HS, Quilez J, Lillehoj EP, et al. Induction of protective immunity against Eimeria tenella, Eimeria maxima, and Eimeria acervulina infections using dendritic cell-derived exosomes. Infect Immun (2012) 80:1909-16. doi:10.1128/IAI.06413-11

30. Twu O, de Miguel N, Lustig G, Stevens GC, Vashisht AA, Wohlschlegel JA, et al. Trichomonas vaginalis exosomes deliver cargo to host cells and mediate hostratioparasite interactions. PLoS Pathog (2013) 9:e1003482. doi:10.1371/journal. ppat. 1003482

31. Benchimol M. The release of secretory vesicle in encysting Giardia lamblia. FEMS Microbiol Lett (2004) 235:81-7. doi:10.1111/j.1574-6968.2004. tb09570.x

32. Gottig N, Elias EV, Quiroga R, Nores MJ, Solari AJ, Touz MC, et al. Active and passive mechanisms drive secretory granule biogenesis during differentiation of the intestinal parasite Giardia lamblia. J Biol Chem (2006) 281:18156-66. doi:10.1074/jbc.M602081200
33. Eger A, Kirch A, Manfras B, Kern P, Schulz-Key H, Soboslay PT. Proinflammatory (IL-1beta, IL-18) cytokines and IL- 8 chemokine release by PBMC in response to Echinococcus multilocularis metacestode vesicles. Parasite Immunol (2003) 25:103-5. doi:10.1046/j.1365-3024.2003.00601.x

34. Walker M, Baz A, Dematteis S, Stettler M, Gottstein B, Schaller J, et al. Isolation and characterization of a secretory component of Echinococcus multilocularis metacestodes potentially involved in modulating the host-parasite interface. Infect Immun (2004) 72:527-36. doi:10.1128/IAI.72.1.527-536.2004

35. Nono JK, Pletinckx K, Lutz MB, Brehm K. Excretory/secretory-products of Echinococcus multilocularis larvae induce apoptosis and tolerogenic properties in dendritic cells in vitro. PLoS Negl Trop Dis (2012) 6:e1516. doi:10.1371/journal. pntd.0001516

36. Senft AW, Philpott DE, Pelofsky AH. Electron microscope observations of the integument, flame cells, and gut of Schistosoma mansoni. J Parasitol (1961) 47:217-29. doi:10.2307/3275292

37. Marcilla A, Trelis M, Cortes A, Sotillo J, Cantalapiedra F, Minguez MT, et al. Extracellular vesicles from parasitic helminths contain specific excretory/secretory proteins and are internalized in intestinal host cells. PLoS One (2012) 7:e45974. doi:10.1371/journal.pone.0045974

38. Andresen K, Simonsen PE, Andersen BJ, Birch-Andersen A. Echinostoma caproni in mice: shedding of antigens from the surface of an intestinal trematode. Int J Parasitol (1989) 19:111-8. doi:10.1016/0020-7519(89)90028-3

39. Threadgold LT. The ultrastructure of the "cuticle" of Fasciola hepatica. Exp Cell Res (1963) 30:238-42. doi:10.1016/0014-4827(63)90233-7

40. Bernal D, Trelis M, Montaner S, Cantalapiedra F, Galiano A, Hackenberg M, et al. Surface analysis of Dicrocoelium dendriticum. The molecular characterization of exosomes reveals the presence of miRNAs. J Proteomics (2014) 105:232-41. doi:10.1016/j.jprot.2014.02.012

41. Maizels RM, Hewitson JP, Murray J, Harcus YM, Dayer B, Filbey KJ, et al. Immune modulation and modulators in Heligmosomoides polygyrus infection. Exp Parasitol (2012) 132:76-89. doi:10.1016/j.exppara.2011.08.011

42. Silverman JM, Clos J, de'Oliveira CC, Shirvani O, Fang Y, Wang C, et al. An exosome-based secretion pathway is responsible for protein export from Leishmania and communication with macrophages. J Cell Sci (2010) 123:842-52. doi:10.1242/jcs.056465

43. Kalra H, Simpson RJ, Ji H, Aikawa E, Altevogt P, Askenase P, et al. Vesiclepedia: a compendium for extracellular vesicles with continuous community annotation. PLoS Biol (2012) 10:e1001450. doi:10.1371/journal.pbio.1001450

44. Saadatnia G, Golkar M. A review on human toxoplasmosis. Scand J Infect Dis (2012) 44:805-14. doi:10.3109/00365548.2012.693197

45. Carruthers V, Boothroyd JC. Pulling together: an integrated model of Toxoplasma cell invasion. Curr Opin Microbiol (2007) 10:83-9. doi:10.1016/j.mib. 2006.06.017

46. World Health Organization. Prevalence and Incidence of Selected Sexually Transmitted Infections, Chlamydia trachomatis, Neisseria gonorrhoeae, Syphilis, and Trichomonas vaginalis: Methods and Results Used by the WHO to Generate 2005 Estimates. Geneva: World Health Organization (2011).

47. Hotez PJ, Brindley PJ, Bethony JM, King CH, Pearce EJ, Jacobson J. Helminth infections: the great neglected tropical diseases. JClin Invest (2008) 118:1311-21. doi:10.1172/JCI34261

48. Regev-Rudzki N, Wilson DW, Carvalho TG, Sisquella X, Coleman BM, Rug $M$, et al. Cell-cell communication between malaria-infected red blood cells via exosome-like vesicles. Cell (2013) 153:1120-33. doi:10.1016/j.cell.2013. 04.029

49. Torrecilhas AC, Schumacher RI, Alves MJ, Colli W. Vesicles as carriers of virulence factors in parasitic protozoan diseases. Microbes Infect (2012) 14:1465-74. doi:10.1016/j.micinf.2012.07.008

50. Cestari I, Ansa-Addo E, Deolindo P, Inal JM, Ramirez MI. Trypanosoma cruzi immune evasion mediated by host cell-derived microvesicles. J Immunol (2012) 188:1942-52. doi:10.4049/jimmunol.1102053

51. Coakley G, Simbari F, McSorley H, Maizels R, Buck A. Secreted exosomes from Heligmosomoides polygyrus modulate cellular responses of the murine host. J Extracell Vesicles (2014) 3:24214. doi:10.3402/jev.v3.24214

52. Hu G, Gong AY, Roth AL, Huang BQ, Ward HD, Zhu G, et al. Release of luminal exosomes contributes to TLR4-mediated epithelial antimicrobial defense. PLoS Pathog (2013) 9:e1003261. doi:10.1371/journal.ppat.1003261

53. Tzipori S, Widmer G. The biology of Cryptosporidium. Contrib Microbiol (2000) 6:1-32. doi:10.1159/000060370 
54. O'Hara SP, Chen XM. The cell biology of Cryptosporidium infection. Microbes Infect (2011) 13:721-30. doi:10.1016/j.micinf.2011.03.008

55. Hassani K, Shio MT, Martel C, Faubert D, Olivier M. Absence of metalloprotease GP63 alters the protein content of Leishmania exosomes. PLoS One (2014) 9:e95007. doi:10.1371/journal.pone.0095007

56. Maizels RM, Hewitson JP, Smith KA. Susceptibility and immunity to helminth parasites. Curr Opin Immunol (2012) 24:459-66. doi:10.1016/j.coi.2012.06.003

57. Hewitson JP, Grainger JR, Maizels RM. Helminth immunoregulation: the role of parasite secreted proteins in modulating host immunity. Mol Biochem Parasitol (2009) 167:1-11. doi:10.1016/j.molbiopara.2009.04.008

58. McSorley HJ, Maizels RM. Helminth infections and host immune regulation. Clin Microbiol Rev (2012) 25:585-608. doi:10.1128/CMR.05040-11

59. Zaccone P, Cooke A. Vaccine against autoimmune disease: can helminths or their products provide a therapy? Curr Opin Immunol (2013) 25:418-23. doi:10.1016/j.coi.2013.02.006

60. Wolff MJ, Broadhurst MJ, Loke P. Helminthic therapy: improving mucosal barrier function. Trends Parasitol (2012) 28:187-94. doi:10.1016/j.pt.2012.02.008

61. Johnston CJ, McSorley HJ, Anderton SM, Wigmore SJ, Maizels RM. Helminths and immunological tolerance. Transplantation (2014) 97:127-32. doi:10.1097/ TP.0b013e3182a53f59

62. Thomas PG, Carter MR, Atochina O, Da'Dara AA, Piskorska D, McGuire E, et al. Maturation of dendritic cell 2 phenotype by a helminth glycan uses a toll-like receptor 4-dependent mechanism. J Immunol (2003) 171:5837-41. doi:10.4049/jimmunol.171.11.5837

63. Vukman KV, Adams PN, Metz M, Maurer M, O’Neill SM. Fasciola hepatica tegumental coat impairs mast cells' ability to drive Th1 immune responses. JImmunol (2013) 190:2873-9. doi:10.4049/jimmunol.1203011

64. Dalton JP, Robinson MW, Mulcahy G, O’Neill SM, Donnelly S. Immunomodulatory molecules of Fasciola hepatica: candidates for both vaccine and immunotherapeutic development. Vet Parasitol (2013) 195:272-85. doi:10. 1016/j.vetpar.2013.04.008

65. Robinson MW, Donnelly S, Hutchinson AT, To J, Taylor NL, Norton RS, et al. A family of helminth molecules that modulate innate cell responses via molecular mimicry of host antimicrobial peptides. PLoS Pathog (2011) 7:e1002042. doi:10.1371/journal.ppat.1002042

66. Robinson MW, Alvarado R, To J, Hutchinson AT, Dowdell SN, Lund M, et al. A helminth cathelicidin-like protein suppresses antigen processing and presentation in macrophages via inhibition of lysosomal vATPase. FASEB J (2012) 26:4614-27. doi:10.1096/fj.12-213876

67. Martinez-Sernandez V, Mezo M, Gonzalez-Warleta M, Perteguer MJ, Muino L, Guitian E, et al. The MF6p/FhHDM-1 major antigen secreted by the trematode parasite Fasciola hepatica is a heme-binding protein. J Biol Chem (2014) 289:1441-56. doi:10.1074/jbc.M113.499517

68. Lund ME, O'Brien BA, Hutchinson AT, Robinson MW, Simpson AM, Dalton JP, et al. Secreted proteins from the helminth Fasciola hepatica inhibit the initiation of autoreactive T cell responses and prevent diabetes in the NOD mouse. PLoS One (2014) 9:e86289. doi:10.1371/journal.pone.0086289

Conflict of Interest Statement: The authors declare that the research was conducted in the absence of any commercial or financial relationships that could be construed as a potential conflict of interest.

Received: 15 May 2014; accepted: 24 August 2014; published online: 08 September 2014. Citation: Montaner S, Galiano A, Trelis M, Martin-Jaular L, del Portillo HA, Bernal D and Marcilla A (2014) The role of extracellular vesicles in modulating the host immune response during parasitic infections. Front. Immunol. 5:433. doi: 10.3389/fimmu.2014.00433

This article was submitted to Immunotherapies and Vaccines, a section of the journal Frontiers in Immunology.

Copyright (c) 2014 Montaner, Galiano, Trelis, Martin-Jaular, del Portillo, Bernal and Marcilla. This is an open-access article distributed under the terms of the Creative Commons Attribution License (CC BY). The use, distribution or reproduction in other forums is permitted, provided the original author(s) or licensor are credited and that the original publication in this journal is cited, in accordance with accepted academic practice. No use, distribution or reproduction is permitted which does not comply with these terms. 